\title{
Changing Indications for Keratoplasty
}

\author{
R. J. MORRIS and A. K. BATES \\ London
}

\begin{abstract}
Summary
The indications for keratoplasty at Moorfields Eye Hospital were reviewed for the period 1985-1987. There were 500 consecutive cases of which 472 underwent penetrating keratoplasty and 28 lamellar keratoplasty; of the grafts performed 31 were acute procedures. The most common indication for penetrating keratoplasty was keratoconus accounting for $\mathbf{3 4 . 2} \%$ of cases and for lamellar keratoplasty corneal dystrophies accounting for $28.6 \%$ of cases. This contrasts with other studies which have found pseudophakic bullous keratopathy to be the leading indication for penetrating keratoplasty. Possible reasons for this difference are discussed and the current series is compared to two previous series presented from this hospital in 1959 and 1974.
\end{abstract}

With the increasing popularity of intra-ocular lens implantation, it has been stated in the literature that pseudophakic bullous keratopathy is now the most common indication for penetrating keratoplasty. However there are few recently reported series to support this contention.

The indications for corneal grafting at Moorfields Eye Hospital were reviewed in $1959^{1}$ and $1974^{2}$. In order to examine how these indications have changed and in particular to evaluate the impact of pseudophakic bullous keratopathy we have studied consecutive corneal grafts performed here during the last three years.

\section{Method}

We have retrospectively reviewed notes of patients undergoing both penetrating and lamellar keratoplasty at Moorfields Eye Hospital from January 1985 to December 1987. These were analysed with respect to age, sex, primary pathology, secondary pathology in cases of regrafting, previous ophthalmic history and ancillary surgery performed at the time of keratoplasty.

The primary pathology for which keratoplasty was performed was determined from clinical diagnosis and where necessary by corroboration with histopathological findings.

\section{Results}

From January 1985 to December 1987, 500 consecutive cases were reviewed of whom 472 underwent penetrating keratoplasty and 28 lamellar keratoplasty; of the grafts performed 31 were acute procedures, the remainder elective.

The indications for corneal grafting are shown in Table I as percentage of total cases and number of grafts performed.

Those designated 'other indications' included corneal leukoma, mesodermal dysgeneses, corneal inlay surgery and chemical burns.

Keratoconus was the most frequent indication for penetrating keratoplasty. There was an equal incidence of right and left eyes 
Table I Indications for keratoplasty 1985-1987

\begin{tabular}{lcc}
\hline & \% of cases & No. of cases \\
\hline Keratoconus & 34.2 & $(171)$ \\
Regrafts & 17.2 & $(86)$ \\
Bullous keratopathy & 12.6 & $(63)$ \\
Corneal dystrophies & 8.6 & $(43)$ \\
Fuchs' dystrophy & 6.2 & $(31)$ \\
Interstitial keratitis & 4.0 & $(20)$ \\
Viral keratitis & 3.2 & $(16)$ \\
Suppurative keratitis & 3.0 & $(15)$ \\
Corneal degeneration & 2.2 & $(11)$ \\
Other indications & 2.2 & $(11)$ \\
Trauma & 2.0 & $(10)$ \\
Corneal scarring & 2.0 & $(10)$ \\
Limbal lesions & 1.4 & $(7)$ \\
Melting disorders & 1.2 & $(6)$ \\
\hline
\end{tabular}

operated upon and the age distribution at keratoplasty is shown in Fig. 1. The mean age of these patients was 27.8 years, compared to 68.5 years for those undergoing penetrating keratoplasty for bullous keratopathy, of all causes, and 71.0 years for those with Fuchs' dystrophy.

In patients undergoing regrafting the primary pathology which necessitated the initial corneal graft is shown in Table II. The subsequent fate of that graft is shown in Table III. In the vast majority of cases the graft had decompensated, in others there had been recurrence of dystrophy, infective keratitis, or corneal melting.

The diagnosis in patients undergoing surgery for corneal dystrophies is shown in Table IV. Amongst those with Fuch's dystrophy, who were grouped separately, 23 cases $(74 \%)$ also underwent cataract extraction at the time of keratoplasty.

Patients with bullous keratopathy were divided into those who were aphakic (44\%) those who were pseudophakic $(40 \%)$ and other cases $(16 \%)$. Of those in the last group three cases followed angle closure glaucoma and four cases of congenital glaucoma or birth trauma.

In patients with bullous keratopathy after cataract extraction the mean interval between the initial surgery and keratoplasty was 6.7 years for aphakic patients and 5.4 years for pseudophakic patients. In patients with pseudophakic bullous keratopathy the type of intraocular lens in situ was iris-fixated in 16 cases, anterior chamber in two cases and posterior chamber in seven cases.

At the time of grafting, the intraocular lens was removed in eight cases (seven iris-fixated lenses and one posterior chamber lens). After removal of the lens an anterior chamber lens was re-implanted in four cases.

Amongst the patients with aphakic bullous keratopathy, $13(46 \%)$ had some other ocular pathology: angle-closure glaucoma in five cases, surgery for primary open angle glaucoma in three cases, retinal detachment surgery in two cases, suppurative keratitis in two cases and trauma in two cases (one patient developed suppurative keratitis and underwent glaucoma surgery).

Lamellar keratoplasties were performed in 28 cases and the indications are shown in Table V. Corneal dystrophies were the most common indication followed by band keratopathy.

The number of grafts performed acutely was 31 or $6.2 \%$, the indications for which are shown in Table VI. Of those cases of suppurative keratitis, four were due to Acanthamoeba.

\section{Discussion}

In our series keratoconus was by far the most common indication for keratoplasty. After this, of similar frequency were regrafts, bullous keratopathy and corneal dystrophies.

Comparison of this series with those reported from this hospital in $1959^{1}$ and $1974^{2}$ show a number of interesting trends. Interstitial keratitis and viral keratitis have progressively declined in importance throughout the series, whilst keratoconus emerged as the most common indication by 1974 . Corneal

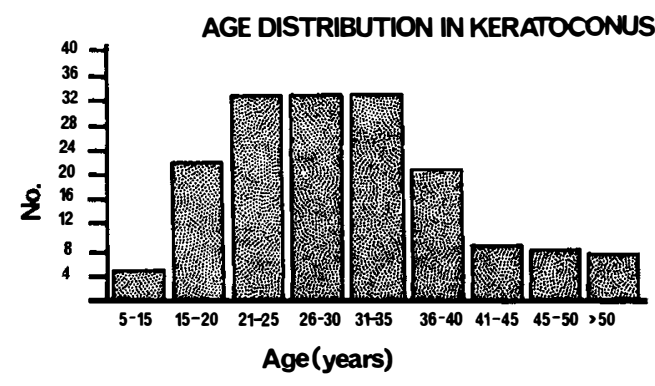

Fig. 1. 
Table II Refgrafts: indications for initial keratoplasty

\begin{tabular}{lc}
\hline & No. of cases \\
\hline Viral keratitis & 19 \\
Corneal dystrophy & 15 \\
Keratoconus & 14 \\
Bullous keratopathy & 8 \\
Suppurative keratitis & 8 \\
Glaucoma (angle closure and & \\
$\quad$ congenital) & 6 \\
Trauma & 3 \\
Scarring & 3 \\
Interstitial keratitis & 3 \\
Fuchs' dystrophy & 2 \\
Corneal melt & 2 \\
Rieger's syndrome & 1 \\
Rosacea keratitis & 1 \\
Phlyctenular keratitis & 1 \\
\hline
\end{tabular}

Table III Regrafts: indications for regraft

\begin{tabular}{lc}
\hline & No. of cases \\
\hline Decompensated graft & 60 \\
Recurrent dystrophy & 6 \\
Suppurative keratitis & 6 \\
Primary graft failure & 5 \\
Corneal melt & 4 \\
Recurrent Herpes Simplex keratitis & 3 \\
Astigmatism & 2 \\
\hline
\end{tabular}

dystrophies have also gradually increased in importance, to the extent that when Fuchs' dystrophy is included, they now constitute the second most important indication for primary grafting with $15 \%$ of cases. Furthermore $29 \%$ of all cases of lamellar keratoplasty performed were for corneal dystrophies.

Many authors express the view that aphakic and particularly pseudophakic bullous keratopathy is now the most common indication for keratoplasty. ${ }^{3.4 .5}$ Smith et al., ${ }^{3}$ found aphakic bullous keratopathy (with and without intraocular lenses) to account for $29.7 \%$ of the corneal grafts performed between 1974 and 1978 and Robin et al., ${ }^{4} 28.4 \%$ of those between 1979 and 1983. In our series such cases constituted only $10.6 \%$ of the total, despite the fact that 2000 cataract extractions are performed annually.

This discrepancy may be explained by a number of factors. Foremost is probably that neither iris-fixated nor anterior chamber intraocular lenses have routinely been implanted after cataract surgery in this hos- pital. Both are believed to be associated with a higher incidence of corneal decompensation than posterior chamber lenses. ${ }^{6.7 .8 .9}$ Indeed this point is supported by the finding that amongst our patients with pseudophakic bullous keratopathy 16 of the 23 had iris-fixated lenses in situ. These patients were almost exclusively referred to this hospital from elsewhere.

Other factors may explain the lower incidence of pseudophakic bullous keratopathy in our series. It is possible that, since patients in this country have mean age in excess of 70 years at the time of cataract surgery ${ }^{10}$ and therefore an average age at grafting of 76 years, surgeons may prefer to perform cataract extraction on the fellow eye rather than penetrating keratoplasty in order to effect visual improvement. Alternatively, it may be that since widespread implantation of intraocular lenses began later in this country than in the United States, pseudophakic bullous keratopathy will yet show a dramatic rise in importance as an indication for keratoplasty in this country in the next few years. However from the delay observed, in this and other series," between cataract surgery and keratoplasty (mean 6.1 years) were this the case one would expect the rising incidence of keratoplasty for this condition to have become manifest by this time.

The frequency of pseudophakic bullous keratopathy itself is likely to be affected by the increasing trend for extracapsular cataract extraction and posterior chamber lens implantation and also by the increased awareness of the importance of the corneal endothelium and use of viscoelastic substances during cataract surgery to protect it.

In patients with bullous keratopathy unrelated to cataract surgery the most common

Table IV Corneal dystrophies

No. of cases

\begin{tabular}{lr}
\hline Macular & 12 \\
Lattice & 10 \\
Granular & 6 \\
Iridocorneal endothelial syndrome & 6 \\
Congenital hereditary endothelial & 5 \\
Reis-Bücklers' & 2 \\
Unknown & 2 \\
\hline
\end{tabular}


Table V Lamellar grafts: indications

\begin{tabular}{lc}
\hline & No. of cases \\
\hline Corneal dystrophies & 8 \\
Band keratopathy & 4 \\
Keratoconus & 4 \\
Limbal dermoid & 3 \\
Pterygium & 2 \\
Scarring & 2 \\
Others & 5 \\
\hline
\end{tabular}

Table VI Acute penetrating keratoplasties: indications

No. of cases
Suppurative keratitis

Corneal melting

Herpes Simplex keratitis

Trauma

Alkali burn
13

11

5

1

1 underlying pathology was acute angle closure glaucoma, known to have a damaging effect on the corneal endothelium. ${ }^{12}$ Angle closure glaucoma also seems to have been a significant factor in the development of bullous keratopathy in a number of aphakic cases in our series.

Regrafting was an important indication for keratoplasty both in our study and that of Robin et al., ${ }^{4}$ accounting for $17.2 \%$ and $15.1 \%$ (1979-1983) of cases respectively. The primary pathology showed a very high incidence of viral keratitis relative to its importance in the overall frequency of indications for keratoplasty, whilst other diagnoses were roughly in the expected ratios from the overall incidence. This feature is present in other series $^{3,4}$ and is thought to reflect the generally less favourable prognosis for keratoplasty in this condition. ${ }^{13}$

Keratoconus was not as important a pathology in regrafted patients in our series, yet it was the most common in that of Smith et al., ${ }^{3}$ This difference is difficult to explain, particularly since the age distribution of patients in the two series is similar (means 27.8 and 34.7 years respectively) and so the incidence of vascularised corneae should not differ significantly between them.

Most cases of regrafting were necessary because of decompensation of the graft, in others there was recurrence of dystrophy, infective keratitis or corneal melting.

The number of grafts performed acutely was $31(6.2 \%)$. Suppurative keratitis and corneal melting were the most common indications and in the former group Acanthamoeba and Pseudomonas were the most commonly identified organisms. There was only one chemical burn in this series, a marked reduction in incidence since with equivalent numbers in 1974 there would have been 35 cases. $^{2}$

In conclusion, our evidence shows that keratoconus remains the most common indication for keratoplasty in this institution and pseudophakic bullous keratopathy is of less importance than suggested in other studies.

\section{References}

1 Ainslie D: A survey of 100 penetrating corneal grafts. Trans Ophthalmol Soc UK 1959, 79: 20920.

2 Ainslie D: Corneal grafting: a comparison between then and now. Trans Ophthalmol Soc UK 1974, 94: $137-43$.

${ }^{3}$ Smith RE, McDonald HR, Nesburn AB et al: Penetrating Keratoplasty: Changing indications, 1947 to 1978. Arch Ophthalmol 1980, 98: 1226-9.

${ }^{4}$ Robin JB, Gindi JJ, Koh K et al: An Update of the Indications for Penetrating Keratoplasty. Arch Ophthalmol 1986, 104: 87-9.

${ }^{5}$ Doughman DJ, Lindstrom RL, Nelson JD: The surgical treatment of external ocular disease. In Easty DL, Smolin G eds. External Eye Diseases. Vol.3. Stoneham, Mass: Butterworths 1985, 32466.

${ }^{6}$ Liesegang T, Bourne W, Ilstrup DM: Short- and Long-term Endothelial Cell Loss Associated with Cataract Extraction and Intraocular Lens Implantation. Am J Ophthalmol 1984, 97: 32-9.

${ }^{7}$ Oxford Cataract Treatment and Evaluation Team, Long-term Corneal Endothelial Cell Loss After Cataract Surgery. Results of a randomised controlled trial. Arch Ophthalmol 1986, 104: 1170-5.

${ }^{8}$ Sugar A, Meyer RF, Heidemann D et al.: Specular Microscopic Follow-up of Corneal Grafts for Pseudophakic Bullous Keratopathy. Ophthalmology 1985, 92: 325-30.

- Glasser D, Matsuda M, Gager W et al.: Corneal Endothelial Morphology after Anterior Chamber Lens Implantation. Arch Ophthalmol 1985, 103: 1347-9.

${ }^{10}$ Oxford Cataract Treatment and Evaluation Team, Cataract Surgery: Interim results and complications of a randomised controlled trial. $\mathrm{Br} J$ Ophthalmol 1986, 70: 402-14.

"Bates AK, Cheng H, Hiorns RW: Pseudophakic 
bullous keratopathy: relationship with endothelial cell density and use of a predictive cell loss model. A preliminary report. Curr Eye Research 1986, 5: 363-6.

${ }^{12}$ Olsen T: The Endothelial Cell Damage in Acute Glaucoma. On the corneal thickness response to intraocular pressure. Acta Ophthalmol 1980, 58: 257-66.

${ }^{13}$ Cobo LM, Coster DJ, Rice NSC et al.: Prognosis and Management of Corneal Transplantation for Herpetic Keratitis. Arch Ophthalmol 1980, 98: $1755-9$. 